'Medicines for Children' before. The majority of adults visit their GP or the NHS choices website (53 counts for both), and adolescents ask friends and family (22) for medication information. Overall participants were happy with the leaflet, with $71.2 \%$ of adults and $54 \%$ of adolescents wanting no changes. Most adolescents (75.7\%) said no to separate leaflets, and were happy reading adult leaflets. A Chi-squared analysis was applied to the recall questions to determine if the correct responses between the two groups were significant for: Ambulance call time: $55.1 \%$ adults answered correctly vs $51.4 \%$ adolescents $(p=0.7016)$. Where to give the Midazolam: $92.1 \%$ adults answered correctly vs $91.9 \%$ adolescents $(p=0.8797)$. Common side effects: $69.9 \%$ adults answered correctly vs $75.7 \%$ adolescents $(p=0.8797)$. These $P$ values show there was no significant difference between adults and adolescents in the information ascertained by the reader.

Conclusion The awareness of MFC was low with many other sources being used for information. Recall of information was good in both groups, showing it's efficacy (perhaps due to the leaflet's 11/12 reading age). Most users, whether adult or adolescent, were content with the leaflet. Adolescents do not want their own leaflets. Medicines for Children's resources would be better spent further promoting public awareness of their existing leaflets rather than developing new teenage adolescent ones.

\section{P022 ANAKINRA FOR USE IN NON- JUVENILE IDIOPATHIC ARTHRITIS (JIA) RELATED HAEMOPHAGOCYTIC LYMPHOHISTIOCYTOSIS (HLH): EVIDENCE BASE AND FUNDING}

Octavio Aragon Cuevas. Alder Hey Children's Hospital and Liverpool John Moores University

10.1136/archdischild-2019-nppc.32

Background Non JIA related HLH is a life-threatening complication that is increasingly recognised in paediatric patients, particularly in those who are unwell in the paediatric intensive care unit (PICU). Untreated or insufficiently treated HLH has a significant mortality rate (up to $53 \%$ ). ${ }^{1}$

Aim To review the evidence base for the use of anakinra in paediatric patients with non-JIA HLH refractory to systemic corticosteroids in patients who are not fit for treatment as per HLH 2004 protocol.

Methods A PubMed search with words 'anakinra' and 'hemophagocytic lymphohistiocytosis' was carried out on July 2018 to find out the evidence base with regards to the use of anakinra in non-JIA related HLH. Any published peer reviewed clinical studies or trials (including but not limited to retrospective or prospective controlled trials, comparative studies and observational/cohort studies) were considered. Case reports and series were considered if better evidence studies were not available. A recent case study from a tertiary paediatric centre will be used to illustrate the pathway followed to diagnose non-JIA related HLH and funding options.

Results Although a protocol exists for primary HLH treatment (HLH 2004), including chemotherapy and stem cell transplantation, ${ }^{2}$ there is no consensus on how to treat secondary HLH. The literature mainly showed case reports and small case series, ${ }^{3}$ describing the use of anakinra collectively for 35 patients (median age 14 to 48 years) who met the HLH 2004 diagnostic criteria with an overall survival rate of up to $88 \%$ at time of discharge from the PICU3. Anakinra was used at standard doses always in combination with corticosteroids. Some patients also received intravenous immunoglobulin (IVIG) and ciclosporin at the discretion of the medical teams. Conclusion The evidence for use of anakinra in non JIA secondary HLH is limited to retrospective observational studies and mostly restricted to adult populations. Despite this caveat, these studies have demonstrated that anakinra therapy alongside other non-etoposide immunomodulatory therapies is associated with an improvement in short term survival. In patients with multi-organ dysfunction, who are too unstable to receive the existing etoposide based HLH-2004 treatment regimen due to concerns regarding significant treatment toxicity, personalised non-etoposide therapies including dexamethasone, IVIG, ciclosporin and anakinra may be better tolerated and provide a bridge to future more standardised treatment. Evidence to date shows that relapse of secondary HLH is possible with ciclosporin therapy. In JIA related HLH, anakinra was considered better than ciclosporin at inducing remission and having a lower incidence of adverse effects, ${ }^{4}$ and NHS England granted funding for the treatment based on these findings. The available evidence did not show any serious adverse events related to anakinra.

Recommendations This tertiary centre approved the use of anakinra for this patient and future patients with this indication despite lack of reimbursement from NHS England for the drug. An urgent interim policy review will be put together by a team of the British Society of Paediatric and Adolescent Rheumatology (BSPAR) and presented to the NHS England commissioners to seek funding for anakinra for paediatric patients with this indication

\section{REFERENCES}

1. Miettunen, et al. Successful treatment of severe paediatric rheumatic disease-associated macrophage activation syndrome with interleukin-1 inhibition following conventional immunosuppressive therapy: case series with 12 patients. Rheumatology (Oxford) 2011;50:417-9

2. Henter Jl, Horne A, Arico M, Egeler RM, Filipovich AH, Imashuku S, et al. HLH2004: Diagnostic and therapeutic guidelines for hemophagocytic lymphohistiocytosis. Pediatr Blood Cancer 2007;48:124-31.

3. Kumar B, Aleem S, Saleh H, Petts J, Ballas ZK. A Personalized Diagnostic and Treatment Approach for Macrophage Activation Syndrome and Secondary Hemophagocytic Lymphohistiocytosis in Adults. J Clin Immunol 2017;37:638-643

4. Boom V, et al. Evidence-based diagnosis and treatment of macrophage activation syndrome in systemic juvenile idiopathic arthritis. Pediatr Rheumatol Online J 2015:13:55

\section{P023 IMPROVING PARENTERAL NUTRITION PRESCRIBING}

Amy Phipps, Wendy Saegenschnitter, Lizzie Hutchison, Vanessa McLelland, Sam Whiting, Heather Weerdenburg. Bristol Royal Hospital for Children

\subsection{6/archdischild-2019-nppc.33}

Background Inpatient parenteral nutrition (PN) is historically administered by nurses against a fluid prescription with flow rates for aqueous and lipid phases. The prescription used to order PN from the aseptic unit is held with clinical pharmacists and not kept on the ward. On call doctors who are not familiar with the patient are asked to write the fluid prescription using an insert sheet accompanying the product. This process is fraught with delays and creates extra work for nurses and doctors. Nurses can't plan the optimal time to start $\mathrm{PN}$ in relation to other patient care, PN often starts late and often coincides with the end of shift or handover. Setting up $\mathrm{PN}$ at busy times is recognised within the hospital as a potential contributor towards errors. An audit on the 